\title{
BeppoSAX observations of Seyfert 1s in the Piccinotti sample
}

\section{I. poorly studied sources}

\begin{abstract}
A. Quadrelli, A. Malizia, L. Bassani, and G. Malaguti
IASF/CNR, via Piero Gobetti 101, 40129 Bologna, Italy

Received 16 June 2003 / Accepted 15 September 2003

Abstract. In this work we present the first of two papers devoted to the study of the X-ray spectral characteristics of Seyfert 1 galaxies in the Piccinotti sample. In particular we analyse here the BeppoSAX broad band (0.1-100 keV) data of 4 objects which, despite their X-ray brightness, have been historically poorly studied due to their late identification with an AGN; these are H0111-149 (MKN 1152), H0235-525 (ESO 198-G24), H0557-385 (IRAS F05563-3820) and H1846-786 (IRAS F18389-7834). We have assumed for all the sources a baseline model which includes a power law with an exponential cut-off plus a reflection component and an iron $\mathrm{K}_{\alpha}$ line; we have also searched for the presence of intrinsic absorption and/or a soft excess component. Our analysis indicates the presence of complex absorption in two objects (H0557-385 and H0111-149) best described by a combination of two uniform absorbers, one cold and one warm. Only in one source, H0557-385, a soft excess component has been measured. The primary continuum is best described by a canonical power law $(\Gamma=1.7-2)$ with a high energy cut-off in the range $40-130 \mathrm{keV}$. A cold reflection component is likely present in all sources with values ranging from less than 0.6 to higher than 2. In 3 out of 4 objects we find a cold iron line having equivalent width typical of Seyfert $1 \mathrm{~s}$ $(100-200 \mathrm{eV})$.
\end{abstract}

Key words. X-rays: galaxies - galaxies: Seyfert - galaxies: general

\section{Introduction}

The A2 experiment on the HEAO-1 satellite performed a complete X-ray survey of $8.2 \mathrm{sr}$ of the sky $(65.5 \%$ coverage) at $|b| \geq 20^{\circ}$ in the $2-10 \mathrm{keV}$ band (Piccinotti et al. 1982). Among the 85 sources detected down to a limiting flux of $3.1 \times 10^{-11} \mathrm{erg} \mathrm{cm}^{-2} \mathrm{~s}^{-1}, 36$ were classified as active galactic nuclei (AGN). They form a complete hard X-ray selected sample of nearby AGN consisting of 22 Seyfert galaxies of type $1-1.5,8$ of type $1.8-2$ plus 1 QSO (3C273), 1 Starburst galaxy (M 82) and 4 BL Lac objects. The sample of type 1 Seyferts is big enough to allow some statistical studies of the bulk properties of these type of AGN: by analysing the X-ray spectra of the entire sample we can tackle a number of issues still unsettled, like defining the incidence of soft excesses, the role and type (cold and/or warm) of absorption(s), the parameter space of various spectral components such as the reflection, the high energy cut-off, the photon index and the iron line equivalent width $(E W)$ as well as the presence of correlations between them.

Send offprint requests to: A. Quadrelli, e-mail: quadrelli@bo.iasf.cnr.it
BeppoSAX observations offer in this sense a unique opportunity since they provide broad band data of sufficiently high quality to allow such a study. Of the 21 objects in the sample, BeppoSAX has observed 18 sources over the entire range of the instrument (0.1-200 keV), while one (NGC 3227) has been serendipitously detected by the PDS instrument only.

Here we present the first of two papers devoted to the study of the broad band X-ray spectral characteristics of Seyfert 1 galaxies in the Piccinotti sample: in particular, we analyse 4 objects which, despite their X-ray brightness, have been historically poorly studied due to their late identification with an AGN. These are H0111-149 identified with MKN 1152 at $z=0.0536$ by Turner \& Pounds (1989), H0235-525 identified with ESO 198-G24 at $z=0.045$ by Ward \& Shafer (1988), H0557-385 identified with IRAS F05563-3820 at $z=$ 0.034 by Fairall et al. (1982) and H1846-786 identified with IRAS F18389-7834 at $z=0.084$ by Remillard et al. (1986). $\mathrm{X}$-ray spectra of these objects were obtained for the first time by EXOSAT (Turner \& Pounds 1989), except for H0557-385, since in this case the data were contaminated by the presence of a nearby source (Giommi et al. 1989). The observed spectra were all canonical power laws with evidence of intrinsic 
Table 1. Observation Log.

\begin{tabular}{lcccccccc}
\hline \hline Source & Start date & \multicolumn{3}{c}{$T_{\text {exp }}(\mathrm{Ks})$} & & \multicolumn{3}{c}{ Counts/s } \\
\cline { 3 - 4 } \cline { 7 - 9 } & & LECS & MECS & $P D S$ & & $L E C S$ & MECS & $P D S$ \\
\hline H0235-525 (1) & 2001-Jan.-23 & 56 & 144 & 63 & & $0.13 \pm 0.002$ & $0.20 \pm 0.001$ & $0.32 \pm 0.02$ \\
H0235-525 (2) & 2001-Jul.-05 & 23 & 97 & 44 & & $0.07 \pm 0.002$ & $0.11 \pm 0.001$ & $0.23 \pm 0.03$ \\
H0557-385 (1) & 2000-Dec.-19 & 21 & 29 & 15 & & $0.23 \pm 0.004$ & $0.44 \pm 0.004$ & $0.47 \pm 0.05$ \\
H0557-385 (2) & 2001-Jan.-26 & 4.8 & 14 & 5.8 & & $0.29 \pm 0.009$ & $0.51 \pm 0.006$ & $0.51 \pm 0.06$ \\
H0111-149 & 2001-Jan.-04 & 33 & 79 & 40 & & $0.05 \pm 0.001$ & $0.07 \pm 0.001$ & $0.11 \pm 0.03$ \\
H1846-786 & 2001-Mar.-08 & 85 & 34 & 18 & & $0.08 \pm 0.004$ & $0.11 \pm 0.002$ & $0.21 \pm 0.04$ \\
\hline
\end{tabular}

Table 2. Fluxes.

\begin{tabular}{|c|c|c|c|c|c|}
\hline \multirow[t]{2}{*}{ Source } & \multicolumn{3}{|c|}{ Flux $^{a}$} & \multirow{2}{*}{$\overline{N_{\mathrm{Gal}}^{b}}$} & \multirow[t]{2}{*}{$z$} \\
\hline & $(0.1-2 \mathrm{keV})$ & $(2-10 \mathrm{keV})$ & $(20-100 \mathrm{keV})$ & & \\
\hline H0235-525 (1) & 0.37 & 1.5 & 2.4 & 3.05 & 0.0455 \\
\hline H0235-525 (2) & 0.07 & 0.9 & 2.2 & 3.05 & 0.0455 \\
\hline H0557-385 (1) & 0.8 & 3.6 & 2.8 & 3.98 & 0.0344 \\
\hline H0557-385 (2) & 1.0 & 4.0 & 5.7 & 3.98 & 0.0344 \\
\hline H0111-149 & 0.11 & 0.57 & 0.99 & 1.60 & 0.0527 \\
\hline H1846-786 & 0.38 & 0.76 & - & 9.06 & 0.0740 \\
\hline
\end{tabular}

absorption in one (H0111-149) out of 3 objects. All 4 sources were observed by ASCA but only in the case of H0557-385 the data have been published (Turner et al. 1996).

On top of a canonical AGN spectrum, H0557-38 presented a complex structure below $2 \mathrm{keV}$ indicative of attenuation by an ionized absorber fully or partially covering the source, beyond a neutral absorber fully covering the source; a soft excess component was also evident. Finally an iron K-shell emission line, which appeared to be significantly broad, with an equivalent width of $300 \mathrm{eV}$ was detected. Inspection of the Tartarus database (see http://tartarus.gsfc.nasa.gov) for unpublished observations, confirms EXOSAT findings of a canonical power law in the other 3 objects, the presence of intrinsic absorption in H0111-149 and evidence for an iron line in H0111-149 and H0235-525. So far BeppoSAX data have been published for only one source, i.e. the first of the two pointings performed on H0235-525. Guainazzi (2003) discussed these data in conjunction with an XMM measurement of the source, which had a spectrum characterized by reprocessing features (reflection and iron line) produced in an accretion disk.

At low energies $(0.1-2 \mathrm{keV})$ the entire sample has been studied by Schartel et al. (1997) using all sky survey data from the ROSAT satellite. These authors found that all 4 objects could be fitted with power laws having photon indeces in the range 2.1-3.2 and that only one source (H0557-385) displayed significant absorption.

Finally, Malizia et al. (1999) performing a systematic coverage of the whole Piccinotti sample with BATSE have indicated that all four sources were marginally detected (about $3 \sigma$ level) at high energies with a $20-100 \mathrm{keV}$ flux in the range $4-6 \times 10^{-11} \mathrm{erg} \mathrm{cm}^{-2} \mathrm{~s}^{-1}$. Long term light curves in the $2-10 \mathrm{keV}$ band indicate substantial flux variations in all
4 sources over timescales of months/years and so BeppoSAX very likely sampled a particular state of each source.

\section{Data analysis}

The BeppoSAX observation Log is shown in Table 1 which lists for each source the observation date, the exposure time and the count rate in each of the narrow field instruments, while other relevant data such as the source redshift, the galactic column density in the direction of each object and the $0.1-2,2-10$ and $20-100 \mathrm{keV}$ fluxes relative to the best fit model are listed in Table 2.

Standard data reduction was performed using the software package "SAXDAS" (see http://www.asdc.asi.it/ software and the Cookbook for BeppoSAX NFI spectral analysis, Fiore et al. 1998). Data were linearized and cleaned from Earth occultation periods and unwanted period of high particle background (satellite passages through the South Atlantic Anomaly). Data have been accumulated for Earth elevation angles $>5$ degrees and magnetic cut-off rigidity $>6$. For the PDS data we adopted a fine energy and temperature dependent Rise Time selection, which decreases the PDS background by $\sim 40 \%$. This improves the signal to noise ratio of faint sources by about 1.5 (Frontera et al. 1997; Fiore et al. 1998).

\subsection{Imaging analysis}

Inspection of the LECS and MECS images indicates the presence of serendipitous X-ray sources in the field of view of two of our sources: H0111-149 and H0557-385. In the first case, an active galaxy identified in the Simbad catalog as 

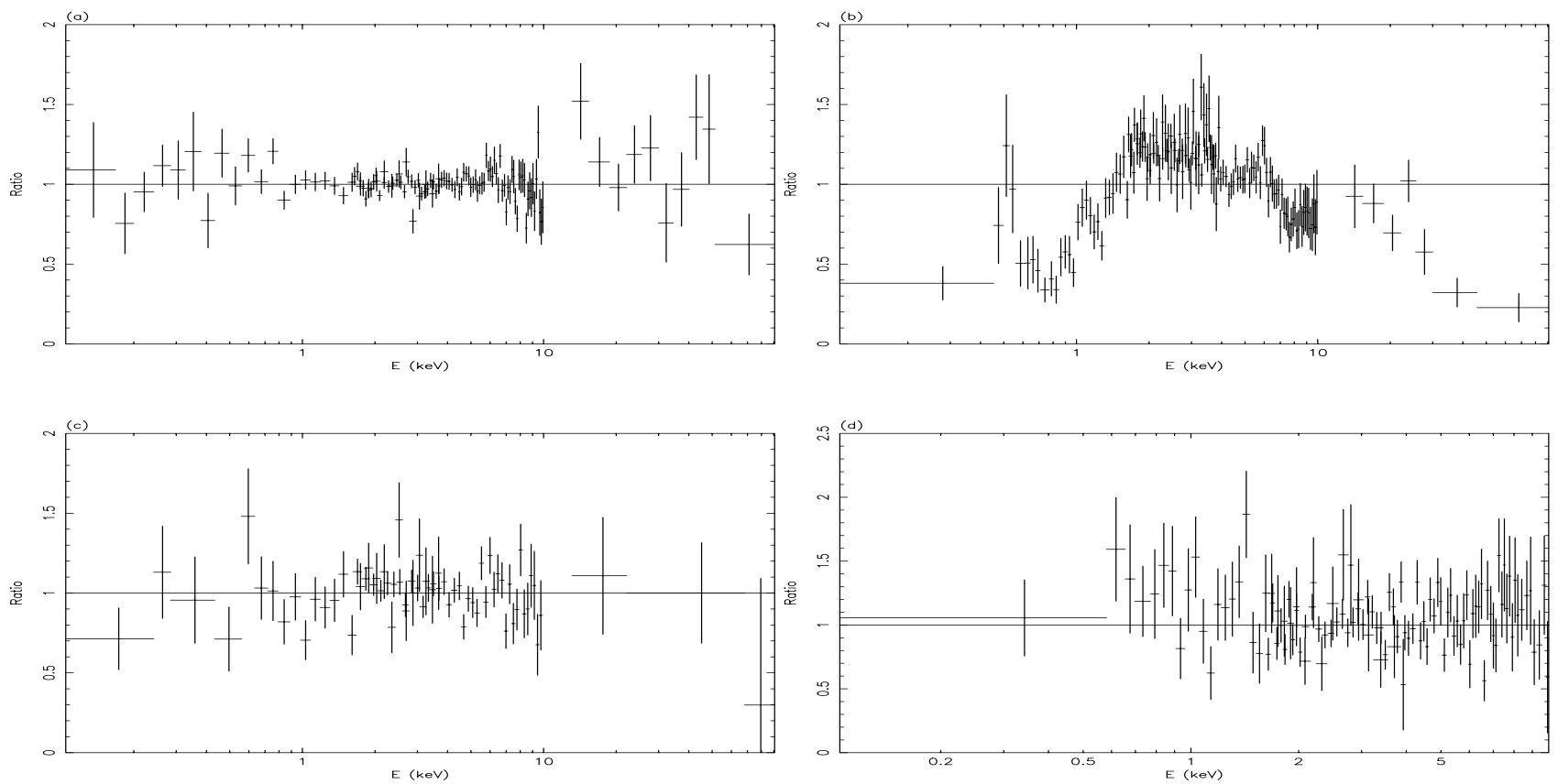

Fig. 1. Data to model ratios of H0235-525 during the second observation a), H0557-385 during the first observation b), H0111-149 c) and H1846-786 d). The fitting model is a simple power law absorbed by the Galactic column density.

EXO 0111.4-1458 (1RPX J01135601442.4) is visible in both LECS and MECS images and located at only 8.1 arcmin from $\mathrm{H} 0111-149$. Its count rate in the $0.1-2 \mathrm{keV}$ is 20 times lower than that of H0111-149, thus excluding contamination in the PDS energy band. In the case of H0557-385 two sources are detected in the LECS image, one of which is also visible in the MECS field of view. The object detected by both instruments is identified with a cataclysmic variable star 30 arcmin away from the target source; the object only seen by the LECS is instead a BL Lac object (EXO055625-3838.6) 25 arcmin from $\mathrm{H} 0557-385$. Both sources have an X-ray spectrum too steep (the star is not detected above $5 \mathrm{keV}$ and the BL Lac above $2 \mathrm{keV}$ ) to be considered as potentially contaminating for the PDS. No other objects, beside the target source is visible in the images of H0235-525 and H1846-786. Despite this, the MECS-PDS cross calibration constant obtained in the fit of H1846-786 suggests the presence of one or more contaminating sources in the PDS observation of this Seyfert; this constant is much higher (2.03-3.85) than the expected value of 0.70-0.95. Within 1 degree of H1846-786, we find a bright Seyfert 1 galaxy (ESO 025-G002, $z=0.028$ ), having a ROSAT flux only 5 times lower than the target object; at least 3 more AGN of type 1 are present in the PDS field of view although they are all much less intense. For this reason, special caution was taken when treating the PDS data of H1846-786 (see Sect. 2.4).

\subsection{Spectral analysis}

Spectral data were extracted from regions centered on each source with a radius of 4 arcmin for LECS and MECS; background spectra were taken from blank sky field regions having the same size in detector coordinates. Source plus background light curves did not show any significant variation and therefore data have been grouped for spectral analysis. Spectral fits were performed using XSPEC 11.2.0 software package and public response matrices as from the 1998 November issue. PI (Pulse Invariant) channels were rebinned in order to have at least 20 counts per bin. This guarantees the use of the $\chi^{2}$ method in determining the best fit parameters, since the distribution in each channel can be considered Gaussian. Constant factors have been introduced in the fitting models in order to take into account the inter-calibration systematic uncertainties between instruments (Fiore et al. 1998). All quoted errors correspond to $90 \%$ confidence level for one interesting parameter $\left(\Delta \chi^{2}=\right.$ 2.71). All models used in what follows contain an additional term to allow for the absorption of X-rays due to our galaxy (see Col. 5 of Table 2). The $N_{\mathrm{H}}$ galactic values, based on $21 \mathrm{~cm}$ radio measurements, were provided by XSPEC.

The data to model ratios assuming a power law absorbed by only a galactic column density are shown in Fig. 1 which highlights spectral differences between sources and extra features to be investigated further. To this end and also to compare our data to previous works, we have assumed for all sources a baseline model which includes: a photon power law (PL) with exponential cut-off $\left[A E^{-\Gamma} \exp \left(-E / E_{\mathrm{f}}\right)\right]$ together with a reflection component $(R=\Omega / 2 \pi)$ from a cold slab isotropically illuminated by the PL photons and subtending a solid angle $\Omega$, with inclination cos $i$ to the line of sight (Magdziarz \& Zdziarski 1995, module PEXRAV) and a Gaussian line in the rest frame of the source to represent the iron $\mathrm{K}_{\alpha}$ fluorescence. In no case we found evidence for a broad line and therefore in the following analysis the line was always assumed to be narrow (sigma $=0$ ). In addition to the Galactic column density we have also considered intrinsic absorption at the source following the results of Perola et al. (2002). Furthermore, for 
Table 3. Results of the spectral analysis.

\begin{tabular}{lccccccc}
\hline \hline Source & $\Gamma$ & $\begin{array}{c}E_{\mathrm{c}} \\
(\mathrm{keV})\end{array}$ & $\begin{array}{c}R \\
(\cos i=0.9)\end{array}$ & $\begin{array}{c}E\left(\mathrm{Fe}_{\mathrm{K} \alpha}\right) \\
(\mathrm{keV})\end{array}$ & $\begin{array}{c}E W \\
(\mathrm{eV})\end{array}$ & $\begin{array}{c}\cos i \\
(R=1)\end{array}$ & $\chi^{2} /$ d.o.f. \\
\hline H0235-525 (1) & $1.74_{-0.03}^{+0.03}$ & $132_{-64}^{+441}$ & $0.49_{-0.34}^{+0.41}$ & $6.35_{-0.22}^{+0.34}$ & $61_{-36}^{+35}$ & $0.25_{-0.11}^{+0.16}$ & $128 / 117$ \\
H0235-525 (2) & $1.72_{-0.04}^{+0.02}$ & $128_{-45}^{+114}$ & $1.50_{-0.32}^{+0.39}$ & $6.68_{-0.32}^{+0.31}$ & $120_{-69}^{+67}$ & $0.90_{-0.25}^{+0.05}$ & $118 / 110$ \\
H0557-385 (1) & $1.92_{-0.03}^{+0.04}$ & $41_{-9}^{+80}$ & $1.66_{-0.36}^{+0.47}$ & $6.17_{-0.14}^{+0.13}$ & $121_{-52}^{+52}$ & $0.90_{-0.13}^{+0.05}$ & $142 / 165$ \\
H0557-385 (2) & $2.02_{-0.04}^{+0.04}$ & $>130$ & $1.34_{-0.41}^{+0.36}$ & $6.49_{-0.34}^{+0.40}$ & $<152$ & $0.89_{-0.46}^{+0.06}$ & $85 / 73$ \\
H0111-149 & $1.71_{-0.10}^{+0.15}$ & - & $<0.57$ & $6.49_{-0.34}^{+0.54}$ & $130_{-98}^{+95}$ & $<0.33$ & $79 / 76$ \\
H1846-786 & $1.92_{-0.04}^{+0.36}$ & - & - & 6.4 (fixed) & $<205$ & - & $160 / 142$ \\
\hline
\end{tabular}

each sample source we have investigated the nature of this intrinsic absorption assuming either a uniform cold absorber, a partial covering absorber (model PCFABS) and/or a uniform warm absorber (model ABSORI). For both reflection and absorption we adopted the element abundances as in Anders \& Grevesse (1993). All fits were performed with cos $i$ fixed to 0.9 $\left(i=30^{\circ}\right)$, i.e. appropriate for Seyfert of type 1 . Alternatively $\cos i$ was left free to vary, while $R$ was fixed equal to 1 . Because the dependence on $\cos i$ of the reflection component shape is modest, all other parameters turned out identical in practice to those obtained with the fixed angle and the differences in $\chi^{2}$ are insignificant.

Finally we investigated the presence of a soft excess in all sources. Some of the best fit parameters relative to adopted baseline model are listed in Table 3 together with the significance of the fit. Absorption and soft excess components when present are discussed in the text. In the following sections results for each individual source are presented and discussed.

\subsection{H0235-525 (ESO 198-G24)}

BeppoSAX-NFIs pointed at H0235-525 twice (January and July) during 2001 and found the source in two different states; consequently the two measurements have been analyzed individually. Fitting each observation with our baseline model, we find compatible parameters for the primary continuum: photon index $\Gamma \sim 1.7$ and cut-off energy $E_{\mathrm{c}} \sim 130 \mathrm{keV}$ (see Table 3 ). No evidence for intrinsic absorption and/or a soft excess component is present in both data sets (see ratio of Fig. 1a). We measure an iron line with high significance ( $>99 \%$ confidence level) but at slightly different energies, $\sim 6.4 \mathrm{keV}$ in the first observation versus $\sim 6.7 \mathrm{keV}$ in the second one. However, when we plot the iron line energy versus normalization for the two measurements, we find that both parameters are compatible within their relative uncertainties. The only parameter which changes with some confidence between the two BeppoSAX observations is the reflection component. This is clearly seen in Fig. 2 where we plot $R$ versus the high energy cut-off: a factor of 3 higher reflection component is present in the second observation when the $2-10 \mathrm{keV}$ flux is also a factor 1.7 lower than in the first measurement (see also Tables 2, 3).

The results obtained for the first observation are fully compatible with the analysis performed by Guainazzi (2003) although we find a slightly lower equivalent width for the iron line $(60 \mathrm{eV}$ compared to $100 \mathrm{eV})$. This author also

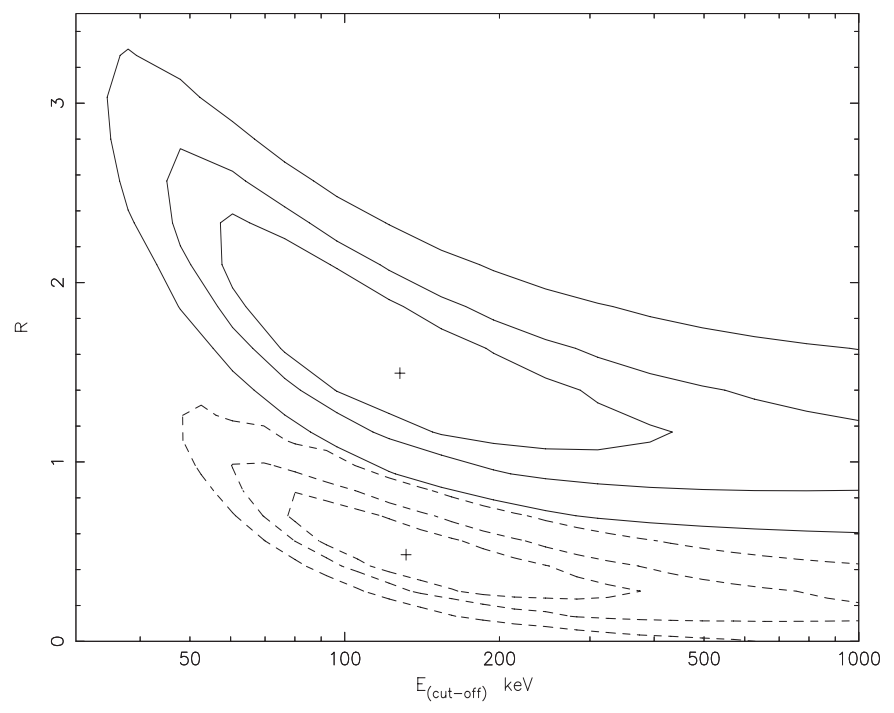

Fig. 2. Confidence contours of the reflection component versus the high energy cut-off in H0235-525: dashed lines are referred to the first observation and solid lines to the second.

studied the time history of the iron line in H0235-525 analysing ASCA, BeppoSAX and XMM observations. He found that during the XMM observation (December 2000) the line was broad and twice as bright as in the BeppoSAX measurement (January 2001); by contrast in the earlier ASCA observation (July 1997) the line was dominated by a remarkably narrow core. This finding was interpreted by the author as indicative of a time lag in the response of the line to the continuum flux in the same way observed by us for the reflection component: in other words, features due to reprocessing emission are delayed in their response to changes in the source intensity. Considering that the time span between our two observations is of the order of 160 days, we can infer an upper limit of $0.14 \mathrm{pc}$ to the distance between the primary continuum emission region and that where reflection occurs. This is compatible with the reprocessing region being localized in the most external part of an accretion disk, in the broad line region or even in a molecular torus.

Combining our results with those of Guainazzi (2003) suggests that the last two sites are more likely to be the region where reprocessing occur. 


\subsection{H0557-385 (IRAS F05563-3820)}

Also H0557-385 has been observed twice by BeppoSAX (December 2000 and January 2001) and found at a similar flux level below $10 \mathrm{keV}$; above this energy the flux changed by about a factor of 2 in just over a month. Therefore the two observations were analyzed individually to search for possible spectral differences particularly at high energies. It is evident that the simple power law model is significantly inadequate to fit the data (see Fig. 1b). But this is hardly surprising giving that the spectrum below $2 \mathrm{keV}$ is highly complex as already shown by ASCA (Turner et al. 1996). This source requires both intrinsic absorption and a soft excess component. The absorber is complex with a cold and a warm column density, both fully covering the source. The cold absorber is of the order of $1.1 \pm 0.3 \times 10^{21} \mathrm{~cm}^{-2}$ in both observations while the warm absorber is $N_{\mathrm{H}^{*}}=8.6_{-0.9}^{+1.2} \times 10^{21} \mathrm{~cm}^{-2}$ with $\xi=5.9_{-4.8}^{+7.8} \mathrm{erg} \mathrm{cm} \mathrm{s}^{-1}$ in the first observation and $N_{\mathrm{H}^{*}}=6.5_{-0.9}^{+1.1} \times 10^{21} \mathrm{~cm}^{-2}$ with $\xi=4.3_{-3.2}^{+4.5} \mathrm{erg} \mathrm{cm} \mathrm{s}^{-1}$ in the second one. Despite the introduction of this complex absorption component, the fit with our baseline model still leaves some residuals below $1 \mathrm{keV}$, strongly indicating the presence of a soft excess component. Following previous ASCA results, we assumed that this soft component maybe due to emission from a hot plasma and parameterize it using a standard Raymond-Smith model. The temperature of the plasma is found to be $0.25 \pm 0.2 \mathrm{keV}$, in agreement with what already observed with ASCA by Turner et al. (1996).

The primary power law is canonical $(\Gamma=1.9-2)$ in both observations with a high energy cut-off increasing from the first $\left(E_{\mathrm{c}_{1}}=41 \mathrm{keV}\right)$ to the second pointing $\left(E_{\mathrm{c}_{2}}>130 \mathrm{keV}\right)$. Inspection of the contours of the cut-off energy versus the power law photon index for the two observations, indicates that the values of $E_{\mathrm{c}}$ are barely compatible at the $90 \%$ confidence level. This change in the high energy cut-off energy also explains the difference in flux above $10 \mathrm{keV}$. An iron line is detected in each measurement with high significance ( $\sim 99 \%$ confidence level): the line is compatible with being neutral and has an equivalent width value typical of Seyfert 1 galaxies $(E W=120-150 \mathrm{eV})$. The reflection is quite strong $(R>1)$ with values compatible between the two observations.

\subsection{H0111-149 (MKN 1152)}

Although marginally evident in the ratio reported in Fig. 1c, H0111-149 requires absorption in excess to the galactic value at the $90 \%$ confidence level: an ionized absorber $\left(N_{\mathrm{H}}=\right.$ $\left.1.61_{-0.47}^{+0.55} \times 10^{21} \mathrm{~cm}^{-2}, \xi=1.12_{-0.68}^{+1.68} \mathrm{erg} \mathrm{cm} \mathrm{s}^{-1}\right)$ or a cold one partially covering the source $\left(N_{\mathrm{H}}=4.2_{-1.0}^{+1.0} \times 10^{21} \mathrm{~cm}^{-2}, C_{\mathrm{f}}=\right.$ $\left.0.40_{-0.16}^{+0.30 \%}\right)$ fit equally well the data $\left(\chi_{\text {warm }}^{2}=81\right.$ for 77 d.o.f. versus $\chi_{\text {cold }}^{2}=82$ for 77 d.o.f.). If instead we introduce in the fits both a cold and warm absorber fully covering the source as done in H0557-525 and used by Perola et al. (2002), the fit is slightly better $\left(\chi^{2}=79.3\right.$ for 76 d.o.f.) and the values for the cold absorber is $N_{\mathrm{H}}=1.19_{-0.59}^{+0.81} \times 10^{20} \mathrm{~cm}^{-2}$ while the warm one has $N_{\mathrm{H}}=2.66_{-2.49}^{+1.17} \times 10^{21} \mathrm{at} \mathrm{cm}^{-2}$ and $\xi=20_{-14}^{+34} \mathrm{erg} \mathrm{cm} \mathrm{s}^{-1}$. In Table 3 we report the best fit parameters relative to this last case. No soft excess is required by the broad band fit. The primary power law is canonical $\left(\Gamma=1.71_{-0.10}^{+0.15}\right)$ and a neutral iron line at $E_{\text {line }}=6.5 \mathrm{keV}$ is detected with high confidence (90\%) with an $E W=125 \mathrm{eV}$.

Unfortunately, the high energy data are of too low statistical quality to allow good constrain to be put on the reflection and high energy cut-off; fixing the high energy cut-off at $E_{\mathrm{c}}>100 \mathrm{keV}$, we were able to constrain the reflection to be smaller than 0.6.

\subsection{H1846-786 (IRAS F18389-7834)}

As discussed in Sect. 2.1 the PDS data of H1846-786 could be contaminated by other Seyfert galaxies present in the field of view, therefore we first analysed only the LECS and MECS data. In the $0.1-10 \mathrm{keV}$ band a simple canonical power law is the best fit to the data (see Fig. 1d). This result indicates no need for extra absorption or the presence of a soft excess. Also the presence of an iron line is not statistically required by the data; in any case fixing the line energy at $6.4 \mathrm{keV}$ as expected in the case of the iron $\mathrm{K}_{\alpha}$ fluorescence line, we obtain an upper limit on the equivalent width of $\sim 200 \mathrm{eV}$ again compatible with the values seen in Seyfert 1s.

We also tried to put some constrain on the reflection and high energy cut-off taking into consideration the PDS data and assuming first that non contaminating sources were present in the field of view. In this case we estimate $R$ to be 2.6, while the high energy cut-off cannot be constrained. On the other hand, in a more realistic case, we should consider the contribution of at least an AGN having a 2-10 keV flux 1/5 that of H1846-786, a photon index of $\Gamma=1.9$ and a reflection component $R=1$, to account for contamination in the PDS data. In this case, the value of the reflection component measured in H1846-786 is still greater than 2 and the high energy cut-off remains unconstrained.

\section{Conclusion}

BeppoSAX observations of four Seyfert 1 galaxies of the Piccinotti sample indicate the presence of complex absorption in two objects (H0557-385 and H0111-149): this absorption is best described by the combination of two uniform absorbers, one cold and one warm. Only one object in the sample (H0557-385) requires a soft excess component. The primary continuum is best described in all sources by a canonical power law with a high energy cut-off in the range $40 \mathrm{keV}$ to higher than $130 \mathrm{keV}$. This seems to be indicative of a large dispersion in cut-off energies due to intrinsic differences between sources but also to variations within a single source; at least in one galaxy (H0557-385) we have some evidence for a change in cut-off energy. A cold reflection component is likely present in all sources: here the observed range of values is large too ranging from less than 0.6 to higher than 2 . This may be due to a large dispersion in this parameter over sources and/or to variations in $R$ in the same source as observed in the case of H0235-525. In 3 out of 4 objects we find a cold iron line having equivalent width typical of type 1 objects, i.e. $100-200 \mathrm{eV}$. The iron line $E W$ is in all cases compatible with the observed strength of the reflection component. In a following paper, we 
expect to extend this study to the entire Piccinotti sample of Seyfert 1 galaxies in order to constrain further the parameter space of the various spectral components and to study possible correlation between them.

Acknowledgements. This research has made use of SAXDAS linearized and cleaned event files produced at the BeppoSAX Science Data Center. We are grateful to P. O. Petrucci for useful discussions on $\mathrm{H} 0235-525$.

\section{References}

Anders, E., \& Grevesse, N. 1993, Geochim. Cosmochim. Acta, 53, 197

Fairall, A. P., McHardy, I. M., \& Pye, J. P. 1982, MNRAS, 198, 13

Fiore, F., Guainazzi, M., \& Grandi, P. 1998, BeppoSAX Cookbook
Frontera, F., Costa, E., Dal Fiume, D., et al. 1997, A\&A, 122, 357

Giommi, P., Bevermann, K., Barr, P., et al. 1989, MNRAS, 236, 375

Guainazzi, M. 2003, A\&A, 903, 910

Magdziarz, P., \& Zdziarski, A. 1995, MNRAS, 273, 837

Malizia, A., Bassani, L., Zhang, K. A., et al. 1999, ApJ, 519, 637

Perola, G. C., Matt, G., Cappi, M., et al. 2002, A\&A, 389, 802

Piccinotti, G., Mushotzky, R. F., Boldt, E. A., et al. 1982, ApJ, 253, 485

Remillard, R. A., Bradt, H. V., Buckley, D. A. H., et al. 1986, ApJ, 301, 742

Schartel, N., Schmidt, M., Fink, H. H., Hasinger, G., \& Trümper, J., 1997, A\&A, 320, 696

Turner, T. J., Netzer, H., \& Gearge, I. M. 1996, ApJ, 463, 134

Turner, T. J., \& Pounds, K. A. 1989, MNRAS, 240, 833

Ward, M. J., Elvis, M., Fabiano, G., et al. 1987, ApJ, 315, 74

Ward, M. J., \& Shafer, R. A. 1988, ApJ, 324, 767 